Trigueros, R.; Aguilar-Parra, J.M.; Álvarez, J.F.; Cangas, A.J.; López-Liria, R. (2020). The Effect of the Motivation on the Resilience and Anxiety of the Athlete. Revista Internacional de Medicina y Ciencias de la Actividad Física y el Deporte vol. 20 (77) pp. 73-86 Http://cdeporte.rediris.es/revista/revista77/artefectos1114.htm

DOI: $10.15366 /$ rimcafd2020.77.005

\title{
ORIGINAL
}

\section{EL EFECTO DE LA MOTIVACIÓN SOBRE LA RESILIENCIA Y LA ANSIEDAD DEL DEPORTISTA}

\section{THE EFFECT OF THE MOTIVATION ON THE RESILIENCE AND ANXIETY OF THE ATHLETE}

\author{
Trigueros, R. ${ }^{1}$; Aguilar-Parra, J. M. ${ }^{1}$; Álvarez, J. F. ${ }^{1}$ Cangas, A. J. ${ }^{1}$ y López- \\ Liria, $\mathbf{R}^{2}$ \\ ${ }^{1}$ Departamento de Psicología, Universidad de Almería (España) rtr088@ual.es \\ 2 Departamento de Enfermería, Fisioterapia y Medicina, Universidad de Almería (España) \\ jmaguilar@ual.es; jalvarez@ual.es; ajcangas@ual.es; rll040@ual.es
}

Código Unesco: 610608 Motivación

Clasificación Consejo de Europa / Council of Europe classification: 15.

Psicología del deporte / Sport Psychology

Recibido 23 de marzo de 2018 Received March 23, 2018

Aceptado 12 de agosto de 2019 Accepted August 12, 2019

\section{RESUMEN}

A lo largo de una carrera deportiva suelen acontecer una serie de hechos traumáticos o fracasos deportivos que suponen un punto de inflexión, por ello, resulta necesario analizar la influencia que tiene la motivación del deportista sobre la resiliencia, y esta última, sobre sus niveles de ansiedad. En este estudio participaron 276 voleibolistas con edades entre 18 y 31 años. En este estudio se han realizado varios análisis estadísticos descriptivos, un análisis de fiabilidad de los factores que integran el modelo y un path analysis que explica las relaciones causales entre las variables. Los índices de ajuste de los diversos análisis fueron satisfactorios explicando las relaciones que se establecen entre los factores. En conclusión, la motivación autodeterminada está relacionada de forma positiva con la resiliencia mientras que las formas menos autodeterminadas están relacionadas de forma negativa con ésta. Asimismo, la resiliencia se relaciona de forma negativa con la ansiedad.

PALABRAS CLAVE: Motivación, psicología, ansiedad, cognición, atleta. 


\section{ABSTRACT}

Throughout a sports career, a series of traumatic events or sporting failures often occur, which represent a turning point. Therefore, it is necessary to analyze the influence of motivation of the athlete on resilience, and the latter, on their levels of anxiety. This study included 276 volleyball players between the ages of 18 and 31 . In this study, several descriptive statistical analyzes were carried out, a reliability analysis of the factors that make up the model and a route analysis that explains the causal relationships between the variables. The indexes of adjustment of the articles of analysis were satisfactory explaining the relationships established between the factors. In conclusion, the selfdetermined motivation is related to the positive form with the resilience while the less self-determined forms are related to the negative form with it. Likewise, resilience is negatively related to anxiety.

KEYWORDS: Motivation, psychology, anxiety, cognition, athlete.

\section{INTRODUCCIÓN}

En los últimos años se ha evidenciado la importancia de la motivación dentro del campo de la Psicología del deporte, y sus efectos sobre el rendimiento deportivo (Stoeber, 2011; Zafra, Álvarez, Montero y Redondo, 2009). Sin embargo, apenas se ha profundizado en la influencia de la motivación sobre la capacidad para sobreponerse a las múltiples dificultades a las que se enfrentan los deportistas tanto a nivel psicológico como físico y social a lo largo de sus vidas deportivas (Broche, Diago y Herrera, 2012; McMorris, 2014). Esta capacidad para enfrentarse a los problemas recibe el nombre de resiliencia que constituye un mecanismo que ayuda al deportista a responder de forma positiva ante situaciones de riesgo (eg., lesiones deportivas, fracasos deportivos y problemas de adaptabilidad a los equipos, etc.) que pueden generar importantes sentimientos de estrés, de depresión y ansiedad en el deportista. En este sentido, Forés y Grané (2008), definen la resiliencia como el conjunto de cualidades personales que constituyen la habilidad humana para superar las situaciones adversas y estresantes, a la vez que suponen un crecimiento positivo en el individuo como resultado de la consecución de sus propios desafíos personales y deportivos, contribuyendo, de este modo, al desarrollo de un exhaustivo proceso de adaptación a nivel conductual, afectivo y social.

En las últimas décadas, diversos investigadores se han apoyado en los postulados de la teoría de la autodeterminación (TAD) con el fin de comprender como los diferentes procesos motivacionales en el ámbito del deporte y de la actividad física, influye a nivel cognitivo y afectivo del deportista (Wouters, Van Nimwegen, Van Oostendorp, y Van Der Spek, 2013). Esta teoría propone la existencia de diferentes tipos y niveles de motivación, situándolos en un continuo de autodeterminación y distinguiendo entre motivación interna o externa al sujeto (Brown y Ryan, 2015; Trigueros, Sicilia, Alcaraz y Dumitru, 2017). Es en este contexto donde se acaban ubicando, de menor a mayor grado de 
autodeterminación, la desmotivación, la motivación extrínseca y la motivación intrínseca.

Comenzando por la desmotivación, esta hace referencia a la ausencia total de motivación o bien a la falta de intención a la hora de realizar una determinada actividad. Un ejemplo ilustrativo de esta cuestión se podría encontrar en el caso de aquellos deportistas que, tras haber sufrido una lesión deportiva grave, carecen de la intención de volver a realizar su actividad deportiva habitual (Almeida, Luciano, Lameiras y Buceta, 2014; Berengüí-Gil, Garcés y HidalgoMontesinos, 2013). Después de la desmotivación se encuentra la motivación extrínseca, que hace especial énfasis en el compromiso que adquiere el deportista con respecto a la actividad deportiva, como un medio para lograr algo o bien como un reto de superación, en comparación con los demás, pero nunca como un logro o superación de sí mismo o de la actividad deportiva (Ryan y Deci, 2014). A su vez, dentro de la motivación extrínseca, aparecen tres tipos diferentes de regulación: El primero de ellos es la regulación externa, que hace referencia a la consecución de premios o al reconocimiento de los demás. Después se encuentra la regulación introyectada, que hace hincapié en el proceso de evitar los sentimientos de culpa que pueden surgir cuando el individuo se encuentra practicando deporte (Ryan y Deci, 2014). A continuación se encuentra la regulación interna, que emerge definida por los beneficios que, para su bienestar, encuentra el individuo en aquello que realiza y, por último, aflora la regulación integrada, comúnmente definida por el hecho de que la actividad física está inmersa en el estilo de vida de la persona (Ryan y Deci, 2014). Para finalizar, aparece la llamada motivación intrínseca, que supone el compromiso del sujeto con el deporte, con la única intención de obtener sensaciones de placer y de disfrute, con lo que la actividad física y deportiva se acaba convirtiendo en un fin en sí misma (Amorose, Anderson-Butcher, Newman, Fraina y lachini, 2016; Thøgersen-Ntoumani, Shepherd, Ntoumanis, Wagenmakers y Shaw, 2016). Por tanto, el concepto de motivación intrínseca muestra una cierta predisposición hacia la asimilación, en un intento de alcanzar ciertas cuotas y niveles de dominio, de indagación y de interés, aspectos que acaban resultando necesarios para el adecuado desarrollo cognitivo, social y personal de los individuos según señala Trigueros, et al., (2017).

Es por ello, que a través de esas experiencias positivas generadas por la propia actividad, los deportistas podrían desarrollar por si mismos la resiliencia ya que no es una cualidad psicológica innata, puesto que debe desarrollarse de manera multidisciplinar y a través de la consecución de retos alcanzables, o mediante la superación de hechos estresantes (Ortín-Montero, De la Vega y GosálvezBotella, 2013). Es por este motivo que los deportistas no van a responder de manera similar ante estas situaciones de riesgo, ya que dependiendo del nivel de desarrollo de la conducta resiliente que hayan adquirido, su rendimiento deportivo puede verse afectado en mayor o menor medida. En esta misma línea, las investigaciones realizadas hasta la actualidad siguieren la existencia de algunos elementos que influyen de manera positiva en la conducta resiliente y que, por tanto, ayudan a los deportistas a alcanzar un rendimiento deportivo óptimo (Sánchez, de Pedro, y Izquierdo, 2016). Entre estos elementos cabe destacarla existencia de una personalidad positiva, unos elevados niveles de motivación intrínseca hacia la actividad deportiva, la confianza en sí mismos, una 
fuerte capacidad de confrontación ante los factores estresantes y el apoyo y el reconocimiento de las principales estructuras y organismos y colectivos de índole social (Sarkar y Fletcher, 2014).

Sin embargo, como dijimos al principio apenas existen estudios que hayan analizado el efecto de los procesos emocionales sobre la resiliencia del deportista (García, Molinero, Ruiz, Salguero, De la Vega y Márquez, 2014; García-Mas, Pujals, Fuster-Parra, Núñez y Rubio, 2014; Vitali, Bortoli, Bertinato, Robazza y Schena, 2015). En este sentido, realizado por Adie, Duda y Ntoumanis (2008), en el que se valieron de una muestra de 235 hombres y 189 mujeres con una edad media de 26,8 años, en el que analizaron como la motivación externa se relacionaba con la resiliencia mostrando una relación predictiva negativa y al contrario, la motivación interna se relacionó de forma positiva respecto a la resiliencia. Esto es debido a que los deportistas que tratan de mejorar sus propias capacidades y habilidades tendiendo, a menudo, a superar los retos propios de su actividad deportiva con altas dosis de esfuerzo, constancia y dedicación.

Un estudio realizado por Belem, Caruzzo, Nascimento Junior, Vieira y Vieira (2014) con jugadores profesionales brasileños de vóley playa, se observó que la motivación interna hacia la mejora personal y la confianza en uno mismo actuó como predictores de la resiliencia. En cuanto a la resiliencia, esta actuó como predictor de la lucha contra la adversidad, la motivación hacia el objetivo y el rendimiento.

Otro estudio realizado por Machida, Irwin y Feltz, (2013) realizado a través de entrevistas fenomenológicas semiestructuradas, con 12 jugadores de rugby que padecieron una lesión en la medula espinal. Los resultados mostraron que el desarrollo de la resiliencia es un proceso multifactorial que involucra factores preexistentes y experiencias previas a la adversidad, donde el apoyo social y experiencias previas resultan claves y que la motivación hacia la mejora de uno mismo.

En el mismo sentido, un estudio realizado por Fletcher y Sarkar (2012) a través de una metodología de corte cualitativo a través de un conjunto de entrevistas a 12 deportistas olímpicos, observaron que el desarrollo óptimo del nivel resiliencia era debido a un conjunto de factores psicológicos entre los que se encontraba la autoeficacia, el apoyo social, la personalidad positiva, una alta motivación interna y un nivel optimo de ansiedad.

Es debido a este último estudio el que la resiliencia pueda tener un papel importante sobre la ansiedad del deportista ya que posee un papel relevante sobre el rendimiento del deportista. En este sentido, Giacobbi y Weinberg (2000) definen la ansiedad como un estado emocional que posee manifestaciones relacionadas con la preocupación, nerviosismo y bloqueo, que producen activación del organismo. Sin embargo, la ansiedad no solo es vista desde un punto de vista negativo sino también positivo debido al nivel de activación que puede tener (Curran, Appleton, Hill y Hall, 2011). Este nivel de activación de la ansiedad no es algo estable, sino que varía a lo largo del día o de la situación, de este modo, si el nivel de ansiedad que experimenta la persona es muy bajo 
se relacionará con un rendimiento bajo. Sin embargo, a medida que la activación del deportista aumenta, lo hará también el rendimiento hasta llegar a su nivel óptimo de ejecución, aunque si siguiese aumentado el rendimiento se vería perjudicado (Carson y Collins, 2016; Osborne, Greene y Immel, 2014).

En definitiva, el objetivo del presente estudio es analizar el grado de influencia de cada uno de los tipos motivacionales que aparecen reflejados en la teoría de la autodeterminación (TAD) respecto a los niveles de resiliencia y de ansiedad que se encuentran presentes en los jugadores de voleibol. De este modo, la hipótesis central del estudio parte de la idea de que las motivaciones que poseen un menor nivel de autodeterminación van influir de forma negativa respecto a la resiliencia, mientras que los estilos motivacionales que presentan un mayor nivel de autodeterminación acabarán teniendo una repercusión mucho más positiva respecto a la resiliencia, de modo que, en función del grado o nivel de resiliencia que presente el deportista en cada momento, también será posible predecir el nivel de ansiedad de éste (a mayor nivel de resiliencia menor ansiedad y a menor nivel de resiliencia más ansiedad).

\section{MATERIAL Y MÉTODO}

\section{PARTICIPANTES}

En el presente estudio han tomado parte 276 voleibolistas (147 varones y 129 mujeres), con edades comprendidas entre los 18 y los 31 años $(M=24.9 ; D T=$ 3.31) y pertenecientes a diferentes clubes deportivos de la provincia de Almería. Los criterios de inclusión para participar en la investigación fueron la participación voluntaria y sincera en el estudio, así como la cumplimentación de todos los cuestionarios y la firma del consentimiento informado.

\section{INSTRUMENTOS}

Escala de Resiliencia en el Contexto Deportivo (ERD). Con el fin de medir la resiliencia en el contexto del deporte se utilizó la escala adaptada de (Trigueros, Álvarez, Aguilar-Parra, Alcaraz y Rosado, 2017) a partir de la The Resilience Scale de Wagnild y Young (1993). Este cuestionario está encabezado por la sentencia "Considerando las vivencias deportivas responde tu grado de acuerdo o desacuerdo con las siguientes afirmaciones" y consta de 25 ítems repartidos en dos factores. Diecisiete de ellos miden la competencia personal (eg."Soy disciplinado con el deporte que practico") y ocho la aceptación de uno mismo y de la vida (eg. "Siento que mi vida deportiva tiene sentido"). Los voleibolistas debían indicar su respuesta por medio de una escala tipo Likert que va de 1 (en desacuerdo) a 7 (totalmente de acuerdo).

La Motivación auto-determinada se evaluó a través del Cuestionario de Regulación Conductual en el Deporte (BRSQ, Behavioral Regulation in Sport Questionnaire; Lonsdale, Hodge y Rose, 2008), validado y adaptado al contexto español por Viladrich, Torregrosa y Cruz (2011) y diseñado para evaluar la motivación en la práctica deportiva desde la perspectiva de la TAD SDT. Esta escala se compone de 24 ítems, repartidos de forma equitativa, además de 
utilizarse para evaluar los motivos de participación en el deporte, a partir de seis subescalas que incluyen la motivación intrínseca, la regulación integrada, la regulación identificada, la regulación introyectada, la regulación externa y la desmotivación. Todos los ítems comparten la raíz "Practico este deporte..." seguida del contenido diferencial por subescala (e.g., "porque me gusta"; "porque me siento presionado por los demás para seguir haciéndolo"; "a pesar de que me pregunto por qué continúo"). Los ítems se valoran de 1 (Completamente Falso) a 7 (Completamente Verdadero)

Las puntuaciones obtenidas en los diferentes tipos de motivación se organizaron en tres índices que agrupaban las formas más autodeterminadas (ie., la motivación intrínseca, la regulación integrada y la regulación identificada), las formas menos autodeterminadas (ie., la regulación introyectada y la regulación externa) y, por último, la ausencia de motivación (desmotivación). Dichos índices eran calculados según la puntuación media de cada uno de los índices (Vallerand, 2007).

La Ansiedad Competitiva en deporte fue medida utilizando la versión española de la escala Sport Anxiety Scale-2 (SAS-2; Smith, Smoll, Cumming y Grossbard, 2006; Ramis, Torregrosa, Viladrich y Cruz, 2010). Diseñada con un conjunto de 15 ítems distribuidos en tres factores de 5 ítems cada uno, se compone de una escala para medir la Ansiedad Somática, la Preocupación y la Concentración Alterada. Los participantes respondían a ítems con la raíz "Antes o mientras compito en deportes..." (e.g., "mi cuerpo siente tensión"; "me preocupo de que no jugaré bien"; "es duro para mí centrarme en lo que se supone que debo hacer"). Cada ítem es contestado en una escala de 4 puntos que va desde "en absoluto" y "mucho".

\section{PROCEDIMIENTO}

Con el fin de poder llevar a cabo el estudio se contactó previamente con los diferentes responsables de los clubes deportivos participantes, explicándoles los motivos de la investigación y, por consiguiente, pidiéndoles su autorización para poder administrar los diferentes cuestionarios a los deportistas. Una vez completado este paso, se explicó a los deportistas que estaban participando en una investigación sobre la motivación en el ámbito del deporte, a la vez que se les solicitó su permiso para poder hacer uso de los cuestionarios respondidos, manteniendo, en todo momento, su anonimato. En este momento, tras proporcionarles esta información, se les pidió que quienes quisieran participar firmaran un consentimiento informado. Los cuestionarios fueron cumplimentados antes del entrenamiento, de forma anónima, y no tardaron más de 15 minutos en contestarlos. Este estudio respeta los principios fundamentales establecidos en la declaración de Helsinki, así como los establecidos en el convenio del consejo de Europa relativo a los derechos humanos y la biomedicina; los establecidos en la legislación española en el campo de la bioética. 


\section{ANÁLISIS DE DATOS}

En primer lugar, se calcularon los estadísticos descriptivos y se efectuó un análisis de correlación entre las variables objeto de estudio, para, seguidamente, realizar un análisis de fiabilidad a través del a de Cronbach y finalmente, llevar a cabo el path analysis de las relaciones predictivas hipotetizadas en el modelo. Para testar los efectos de mediación en el modelo se tuvieron en cuenta las premisas establecidas por Baron y Kenny (1986): (1) Correlaciones significativas entre la variable independiente y la dependiente; (2) Correlaciones significativas entre la variable independiente y los mediadores; (3) Correlaciones significativas entre los mediadores y la variable dependiente; (4) La relación significativa previa entre la variable independiente y la dependiente deja de ser significativa cuando las relaciones entre la variable independiente y los mediadores y entre éstos y la dependiente son controladas. Además, se proporcionaron tanto los efectos directos como los efectos indirectos.

El path analysis se realizó utilizando el método de estimación de máxima verosimilitud con el procedimiento de bootstrapping en el paquete estadístico AMOS 19. Este procedimiento puso de manifiesto la robustez de las estimaciones (Byrne, 2001), a pesar de la ausencia de normalidad en el modelo hipotetizado (coeficiente de Mardia $=161.57$ ). Para analizar la bondad de ajuste del modelo se utilizaron los siguientes índices: the coefficient $x 2$, the chi-square to degrees of freedom ratio $(\mathrm{X} 2 / \mathrm{gl})$, the Comparative Fit Index $(\mathrm{CFI})$, the Incremental Fit Index (IFI), the Root Mean Square Error of Approximation (RMSEA) plus its $90 \%$ confidence interval y the Standardized Root Mean Square Residual (SRMR). Por lo general, se consideran aceptables valores de $x 2 / g l$ inferiores a 5 (Bentler, 1989), valores de CFI e IFI iguales o superiores a .90, valores de 0,06 o inferiores para el RMSEA y de 0,08 o inferiores para el SRMR (Hu y Bentler, 1999).

\section{RESULTADOS}

\section{ANÁLISIS PRELIMINAR}

En la Tabla 1 se pueden observar los estadísticos descriptivos, los análisis de estabilidad temporal y las correlaciones existentes entre las variables objeto de estudio. Se puede observar una mayor puntuación media para motivación intrínseca $(M=5,46)$ y una menor puntuación media para desmotivación $(M=$ 1,91).

En el análisis de correlación se pudo visualizar la presencia de una asociación negativa en la motivación autodeterminada con respecto a las motivaciones no autodeterminadas y a la desmotivación, siendo la correlación entre estas dos últimas, positivas. En cuanto a los tipos de motivación, en relación a la resiliencia, se aprecia que la motivación no autodeterminada y la desmotivación se relacionaban de forma negativa con esta variable a un nivel moderado $\mathrm{y}$, sin embargo, esta relación fue positiva entre la motivación autodeterminada y la resiliencia. Por último, tanto la resiliencia como la motivación autodeterminada, se relacionaron de forma negativa respecto a la ansiedad. A su vez, la 
desmotivación y a la motivación no autodeterminada se relacionan con la ansiedad de forma positiva.

Tabla 1.

Estadísticos descriptivos, análisis de fiabilidad y correlaciónes bivariadas entre las variables.

\begin{tabular}{|c|c|c|c|c|c|c|c|c|}
\hline Variables & $M$ & $D T$ & $\alpha$ & 1 & 2 & 3 & 4 & 5 \\
\hline 1. Mot. Autodeterminada & 5.46 & .86 & .81 & & $-.20^{\star *}$ & $-.53^{\star *}$ & $.40^{* *}$ & $-.48^{* *}$ \\
\hline 2. Mot. No autodeterminada & 3.57 & .83 & .77 & & & $.13^{*}$ & $-.37^{\star *}$ & $.42^{* *}$ \\
\hline 3. Desmotivación & 1.91 & .79 & .86 & & & & $-.53^{\star \star}$ & $.18^{*}$ \\
\hline 4. Resiliencia & 5.35 & 1.15 & .92 & & & & & $-.49^{\star \star}$ \\
\hline 5. Ansiedad & 2.23 & .43 & .78 & & & & & \\
\hline
\end{tabular}

\section{PATH ANALYSIS}

Se testó el modelo de relaciones predictivas hipotetizado (Figura 1). El análisis del modelo hipotetizado obtuvo los siguientes índices de ajuste: $X 2(3, N=276)$ $=10,35, \mathrm{p}=0,002 ; \mathrm{x} 2 / \mathrm{gl}=3,45 ; \mathrm{CFI}=0,97 ; \mathrm{IFI}=0,96 ; \mathrm{TLI}=0,97 ; \mathrm{RMSEA}=$ $0,071(95 \% \mathrm{Cl}=0,068-0,083)$; $\mathrm{SRMR}=0,056$. A continuación se describen las relaciones obtenidas atendiendo a las diferentes hipótesis que fueron planteadas en el estudio:

Las relaciones entre la motivación autodeterminada y no autodeterminada fueron negativas $(\beta=-0,38, p=0,006)$, e igualmente negativa respecto a la desmotivación $(\beta=-0,53, p=0,001)$. La relación entre la motivación no autodeterminada y la desmotivación fue positiva $(\beta=0,35, p=.009)$.

Hipótesis 1: La motivación autodeterminada predijo positivamente la resiliencia $(\beta=0,48, p=0,012)$ y de forma negativa la motivación no autodeterminada ( $\beta=$ $-0,39, p=0,021)$ y la desmotivación $(\beta=-0,12, p=0,008)$.

Hipótesis 2: La resiliencia predijo negativamente $(\beta=-0,33, p<0,001)$ la ansiedad.

Hipótesis 3: En la resiliencia mediaron los efectos derivados de la motivación autodeterminada (efecto indirecto: $\beta=0,05, p=0,009$ ) y de la motivación no autodeterminada (efecto indirecto: $\beta=-0,07, p=0,008$ ) sobre la ansiedad. 


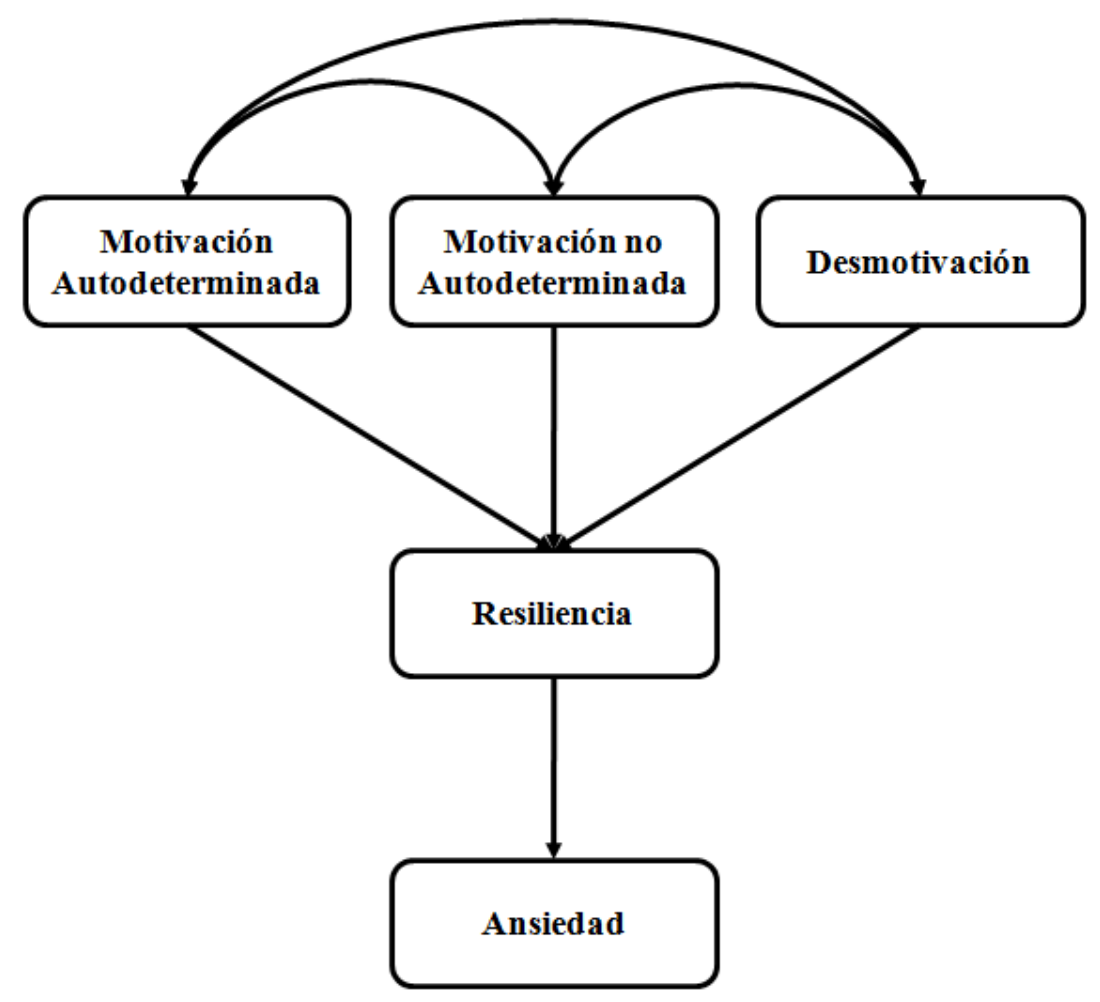

Figura 1. Modelo hipotetizado de la ansiedad en el contexto deportivo

\section{DISCUSIÓN}

En el presente estudio, se contempla por primera vez la resiliencia como un valor predictivo hacia la ansiedad en contexto deportivo, sin olvidarse de que es la primera que se integra en el contexto de la TAD. Estudio previos habían analizado como la motivación autodeterminada predecía el bienestar personal, psicológico y emocional de las personas y como estos bienestares favorecían la adherencia hacia práctica deportiva o de ocio. De este modo este estudio pretende profundizar un poco más en el entendimiento de cómo la resiliencia afecta a los deportistas y esta hacia la ansiedad de los mismos. En este sentido estos resultados dan un apoyo importante a la TAD, clarificando un poco más cómo se podría producir el proceso de transferencia motivacional hacia bienestar emocional de los deportistas.

En línea con la TAD, los resultados mostraron que la motivación autodeterminada predecía positivamente la resiliencia y esta a su vez era predicha de forma negativa por la motivación no autodeterminada y la desmotivación. Además, se hizo visible el efecto mediador que tuvo la resiliencia dentro del modelo (Hipótesis 3). Estos resultados pueden compararse apenas con unos pocos estudios ya que apenas existen investigaciones en el ámbito deportivo que hayan relacionado la motivación con la resiliencia. En este sentido, diversos estudios realizados (eg., Adie, et al., 2008; Belem, et al., 2014; GarcíaMas, et al., 2014; Machida, et al., 2013; Moran, 2016; Vitali, et al., 2015) señalaron que la motivación externa predecía de forma negativa la resiliencia y la motivación interna la predecía de forma positiva. Esto se puede explicar por el hecho de que los deportistas que intentan mejorar sus capacidades y habilidades, con frecuencia, llegan a superar los retos propios de su actividad 
deportiva con elevados y significativos niveles de esfuerzo, de constancia, de dedicación y de sacrificio. Estos aspectos se encuentran ligados a la motivación autodeterminada $y$ en contra de las formas motivacionales menos autodeterminadas de los deportistas.

Los resultados del estudio también mostraron que la resiliencia predecía negativamente la ansiedad. Esta relación, resulta de especial relevancia dado que la resiliencia es un constructo de reciente estudio por la comunidad científica en el ámbito deportivo, por lo cual, hay que recurrir al contexto de la psicología clínica donde existen numerosos estudios que traten estos constructos (eg., Hjemdal, Vogel, Solem, Hagen y Stiles, 2011; Min, Yu, Lee y Chae, 2013), siendo la relación que se establece de predicción negativa entre la resiliencia y la ansiedad. Este resultado puede entenderse por el hecho de que el deportista perciba que el reto al que se enfrenta es alcanzable y la consecución del objetivo u objetivos es materializable, disminuyendo el conflictivo interno y por ende, disminuyendo su ansiedad. Esto pone de manifiesto la importancia que posee la resiliencia en el bienestar emocional de los deportistas, y de la relevancia que tiene el hecho de trabajar con ellos a nivel mental y psicológico para poder enfrentarse a la competición.

\section{CONCLUSIONES}

Este estudio partiendo del enfoque de la TAD ha apoyado sus postulados relacionándola con nuevas variables y mostrando su aplicabilidad a la cultura española. No obstante, en cuanto a los hallazgos existentes en este modelo, resulta necesario recalcar que se trata de un estudio correlacional por lo que no permite extrapolar relaciones de causa-efecto y que los resultados obtenidos podrían ser interpretados de múltiples formas diferentes dependiendo de la interpretación de la persona. Por ello, se tratado de exponer posibilidades y no casualidad, con el fin de poder explicar las relaciones existente entre las variables del estudio. Además, futuros estudios deberían aplicar este modelo en otras disciplinas deportivas ya que únicamente se ha desarrollado con deportistas que juegan al voleibol. Otra limitación ha sido que la muestra no ha sido demasiado amplia, sin embargo el modelo parece mostrar una buena robustez y capacidad de generalización hacia diferentes culturas o edades, el siguiente paso será establecer un estudio etnográfico con el fin de profundizar y entender los aspectos psicológicos del deporte.

\section{REFERENCIAS BIBLIOGRAFICAS}

Amorose, A. J., Anderson-Butcher, D., Newman, T. J., Fraina, M., y lachini, A. (2016). High school athletes' self-determined motivation: The independent and interactive effects of coach, father, and mother autonomy support. Psychology of Sport and Exercise, 26, 1-8. https://doi.org/10.1016/i.psychsport.2016.05.005

Almeida, P., Luciano, L., Lameiras, J., y Buceta, J. M. (2014). Beneficios percibidos de las lesiones deportivas: Estudio cualitativo en futbolistas profesionales y semiprofesionales. Revista de Psicología del Deporte, 23(2), 457-464. 
Adie, J. W., Duda, J. L., y Ntoumanis, N. (2008). Achievement Goals, Competition Appraisals, and the Psychological and Emotional Welfare of Sport Participants. Journal of Sport \& Exercise Psychology, 30(1), 302-322. https://doi.org/10.1123/isep.30.3.302 PMid:18648108

Belem, I. C., Caruzzo, N. M., Nascimento Junior, J. R. A. D., Vieira, J. L. L., y Vieira, L. F. (2014). Impact of coping strategies on resilience of elite beach volleyball athletes. Revista Brasileira de Cineantropometria \& Desempenho Humano, 16(4), 447-455. https://doi.org/10.5007/19800037.2014v16n4p447

Berengüi-Gil, L., Garcés, E.J., y Hidalgo-Montesinos, M.D. (2013). Características psicológicas asociadas a la incidencia de las lesiones en deportistas de modalidades individuales. Anales de Psicología, 29(3), 674-684. http://dx.doi.org/10.6018/analesps.29.3.175811

Broche, Y., Diago, C., y Herrera, L.F. (2012). Características resilientes en jóvenes deportistas y sus pares de la Universidad Central "Marta Abreu" de Las Villas. Revista de la Facultad de Psicología Universidad Cooperativa de Colombia, 8(14), 10-18.

Baron, R. M., y Kenny, D. A. (1986). The moderator-mediator variable distinction in social psychological research: Conceptual, strategic, and statistical considerations. Journal of Personality and Social Psychology, 51(6), 1173. https://doi.org/10.1037/0022-3514.51.6.1173

PMid:3806354

Bentler, P. M. (1989). EQS structural equations program manual. Los Angeles: BMDP Statistical Software.

Brown, K.W., y Ryan, R.M. (2015). A Self-Determination Theory Perspective on Fostering Healthy Self-Regulation From Within and Without. Positive Psychology in Practice: Promoting Human Flourishing in Work, Health, Education, and Everyday Life, Second Edition, 139-158. https://doi.org/10.1002/9781118996874.ch9

Byrne, B.M. (2001). Multivariate applications book series. Structural equation modeling with AMOS: Basic concepts, applications, and programming. Mahwah, NJ: Lawrence Erlbaum Associates, 10.

Carson, H.J., y Collins, D. (2016). The fourth dimension: A motoric perspective on the anxiety-performance relationship. International Review of Sport and Exercise Psychology, 9(1), 1-21. https://doi.org/10.1080/1750984X.2015.1072231 $\quad$ PMid:26692896 PMCid:PMC4662095

Curran, T., Appleton, P. R., Hill, A. P., \& Hall, H. K. (2011). Passion and burnout in elite junior soccer players: The mediating role of self-determined motivation. Psychology of Sport and Exercise, 12(6), 655-661. https://doi.org/10.1016/j.psychsport.2011.06.004

García, X., Molinero, O., Ruiz, F., Salguero, A., De la Vega, R., \& Márquez, S. (2014). La resiliencia en el deporte: fundamentos teóricos, instrumentos de evaluación y revisión de la literatura. Cuadernos de Psicología del Deporte, 14(3), 83-98. $\quad$ https://doi.org/10.4321/S157884232014000300010

García-Mas, A., Pujals, C., Fuster-Parra, P., Núñez, A., \& Rubio, V. (2014). Determinación de las variables psicológicas y deportivas relevantes a las 
lesiones deportivas: Un análisis bayesiano. Revista de Psicología del Deporte, 23(2), 423-429.

Giacobbi Jr, P. R., y Weinberg, R. S. (2000). An examination of coping in sport: Individual trait anxiety differences and situational consistency. The Sport Psychologist, 14(1), 42-62. https://doi.org/10.1123/tsp.14.1.42

Hjemdal, O., Vogel, P. A., Solem, S., Hagen, K., y Stiles, T. C. (2011). The relationship between resilience and levels of anxiety, depression, and obsessive-compulsive symptoms in adolescents. Clinical Psychology \& Psychotherapy, 18(4), 314-321. https://doi.org/10.1002/cpp.719 PMid:20806419

Hu, L., y Bentler, P. M. (1999). Cutoff criteria for fit indexes in covariance structure analysis: Conventional criteria versus new alternatives. Structural Equation Modeling, 6, 1-55. https://doi.org/10.1080/10705519909540118

Fletcher, D. y Sarkar, M. (2012). A grounded theory of psychological resilience in Olympic champions. Psychology of Sport and Exercise, 13(1), 669-678. https://doi.org/10.1016/j.psychsport.2012.04.007

Forés, A. y Grané, J. (2008). La resiliencia. Crecer desde la adversidad. Barcelona: Plataforma.

Lonsdale, C., Hodge, K., y Rose, E.A. (2008). The behavioural regulation in sport questionnaire (BRSQ): Instrument development and initial validity evidence. Journal of Sport and Exercise Psychology, 30, 323-355. https://doi.org/10.1123/isep.30.3.323 PMid:18648109

Machida, M., Irwin, B., y Feltz, D. (2013). Resilience in competitive athletes with spinal cord injury: the role of sport participation. Qualitative Health Research, 23(8), 1054-1065. https://doi.org/10.1177/1049732313493673 PMid:23771633

Min, J. A., Yu, J. J., Lee, C. U., y Chae, J. H. (2013). Cognitive emotion regulation strategies contributing to resilience in patients with depression and/or anxiety disorders. Comprehensive Psychiatry, 54(8), 1190-1197. https://doi.org/10.1016/i.comppsych.2013.05.008 PMid:23806709

McMorris, T. (2014). Acquisition and performance of sport skills. West Sussex: Wiley.

Moran, A. P. (2016). The psychology of concentration in sport performers: A cognitive analysis. Psychology Press. https://doi.org/10.4324/9781315784946

Ortín-Montero, F. J., De la Vega, R., y Gosálvez-Botella, J. (2013). Optimismo, ansiedad-estado y autoconfianza en jóvenes jugadores de balonmano. Anales de Psicología, 29(3), 637-641. https://doi.org/10.6018/analesps.29.3.175751

Osborne, M. S., Greene, D. J., y Immel, D. T. (2014). Managing performance anxiety and improving mental skills in conservatoire students through performance psychology training: a pilot study. Psychology of Well-Being, 4(1), 1-18. https://doi.org/10.1186/s13612-014-0018-3

Ryan, R. M., y Deci, E. (2014). Self-determination theory. In Encyclopedia of Quality of Life and Well-Being Research (pp. 5755-5760). Springer Netherlands. https://doi.org/10.1007/978-94-007-0753-5 2630

Ramis, Y., Torregrosa, M., Viladrich, C., \& Cruz, J. (2010). Adaptación y validación de la versión española de la Escala de Ansiedad Competitiva SAS-2 para deportistas de iniciación. Psicothema, 22(4), 1004-1009 
Sánchez, M. I. S., de Pedro, M. M., y Izquierdo, M. G. (2016). Propiedades psicométricas de la versión española de la escala de resiliencia de 10 ítems de Connor-Davidson (CD-RISC 10) en una muestra multiocupacional. Revista Latinoamericana de Psicología, 48(3), 159-166. https://doi.org/10.1016/j.rlp.2015.09.002

Sarkar, M., y Fletcher, D. (2014). Psychological resilience in sport performers: a review of stressors and protective factors. Journal of Sports Sciences, 32(15), 1419-1434. $\quad$ https://doi.org/10.1080/02640414.2014.901551 PMid:24716648

Smith, R. E., Smoll, F. L., Cumming, S. P., y Grossbard, J. R. (2006). Measurement of multidimensional sport performance anxiety in children and adults: The Sport Anxiety Scale-2. Journal of Sport and Exercise Psychology, 28(4), 479-501. https://doi.org/10.1123/jsep.28.4.479

Stoeber, J. (2011). The dual nature of perfectionism in sports: Relationships with emotion, motivation, and performance. International Review of Sport and Exercise Psychology, 4(2), 128-145. https://doi.org/10.1080/1750984X.2011.604789

Thøgersen-Ntoumani, C., Shepherd, S. O., Ntoumanis, N., Wagenmakers, A. J., \& Shaw, C. S. (2016). Intrinsic motivation in two exercise interventions: Associations with fitness and body composition. Health Psychology, 35(2), 195-198. https://doi.org/10.1037/hea0000260 PMid:26389719

Trigueros, R., Álvarez, J.F., Aguilar-Parra, J.M., Alcaraz, M. y Rosado, A. (2017). Validación y Adaptación Española de la Escala de Resiliencia en el Contexto Deportivo (ERCD). Psychology, Society, \& Education, 9(2), 311324. https://doi.org/10.25115/psye.v9i2.864

Trigueros, R., Sicilia, A., Alcaraz-Ibáñez, M., y Dumitru, D. C. (2017). Adaptación y validación española de la escala revisada del locus percibido de causalidad (PLOC-R) en educación física. Cuadernos de Psicología del Deporte, 17(1), 25-32.

Vallerand, R. J. (2007). A hierarchical model of intrinsic and extrinsic motivation for sport and physical activity. In M. S. Hagger y N. L. D. Chatzisarantis (Eds.), Intrinsic motivation and self-determination in exercise and sport (pp. 255-279, 356-363). Champaign, IL: Human Kinetics.

Viladrich, C., Torregrosa, M., y Cruz, J. (2011). Calidad psicométrica de la adaptación española del Cuestionario de Regulación Conductual en el Deporte. Psicothema, 23(4), 786-794.

Vitali, F., Bortoli, L., Bertinato, L., Robazza, C., y Schena, F. (2015). Motivational climate, resilience, and burnout in youth sport. Sport Sciences for Health, 11(1), 103-108. https://doi.org/10.1007/s11332-014-0214-9

Wagnild, G., y Young, H. (1993). Development and psychometric. Journal of Nursing Measurement, 1(2), 165-17847.

Wouters, P., Van Nimwegen, C., Van Oostendorp, H., y Van Der Spek, E. D. (2013). A meta-analysis of the cognitive and motivational effects of serious games. Journal of Educational Psychology, 105(2), 249. https://doi.org/10.1037/a0031311

Zafra, A. O., Álvarez, M. A., Montero, F. J. O., y Redondo, A. B. (2009). Ansiedad competitiva, percepción de éxito y lesiones: un estudio en futbolistas. Revista Internacional de Medicina y Ciencias de la Actividad Física y del Deporte, 9(33), 51-66. 
Número de citas totales / Total references: 41 (100\%)

Número de citas propias de la revista / Journal's own references: 1 (2,3\%)

Rev.int.med.cienc.act.fís.deporte - vol. 20 - número 77 - ISSN: 1577-0354 